\title{
TEOLOGÍA DE LA VERDAD PRESENTE Y LA MISIÓN ADVENTISTA
}

MG. AlLAN BORNAPÉ, PHD (C)

Universidad Adventista de Chile

Chile 


\title{
Resumen
}

Teología de la verdad presente y la misión adventista. El concepto de verdad presente configura el pensamiento teológico fundamental del adventismo y define su mensaje y misión. A pesar de su enorme valor, no ha recibido la atención necesaria al interior de los círculos teológicos adventistas contemporáneos, incluyendo sus alcances e implicancias para la iglesia. Este artículo busca dilucidar el significado de los componentes de la verdad presente, su origen y desarrollo histórico, y sus bases en la apocalíptica bíblica, para finalmente presentar su diseño teológico. Se concluye que la teología de la verdad presente debería tener un considerable impacto en el quehacer teológico adventista y la misión de la iglesia.

Palabras clave: Verdad presente, teología, apocalíptica, historia, carácter.

\begin{abstract}
Theology of the Present Truth and the Adventist Mission. The concept of the present truth sets up the theological thought which is foundational for the Adventism and defines its message and mission. Despite of its great value, it has not received enough attention within the current Adventist theological circles, including its scopes and implications for the church. This article searches to clarify the meaning of the components of the present truth, its origin and historical development, and its basis on the biblical apocalyptic, in order to finally present its theological design. The article concludes that the theology of the present truth should have a considerable impact in the Adventist theological task and the mission of the church.
\end{abstract}

Keywords: Present truth, theology, apocalyptic, history, character.

Recibido: 04/04/2021

Aceptado: 17/10/2021 
Estrategias para el Cumplimiento de la Misión 19, no. 1 (2021): 5-20

\title{
TEOLOGÍA DE LA VERDAD PRESENTE Y LA MISIÓN ADVENTISTA
}

\author{
MG. Allan BornAPÉ. PHD (C) \\ Universidad Adventista de Chile \\ Chile
}

\begin{abstract}
1. Introducción
Una de las ideas más preciadas para el adventismo es lo que llamamos verdad presente. Básicamente, esta reúne un grupo de verdades bíblicas que constituyen, según creemos como adventistas, los pilares de la fe bíblica, las nociones más fundamentales de las Escrituras. No obstante, para muchos adventistas hoy en día esta terminología parece difusa y algo extraña. Aún los teólogos no están muy involucrados con ella. En este artículo, presento más a fondo solo tres aspectos de este concepto para así observar su relevancia e impacto para la misión de la iglesia.
\end{abstract}

\section{Verdad presente: tiempo y profecía}

El primer aspecto que resulta más evidente sobre la verdad presente es, precisamente, su idea del tiempo: es algo para el presente. ¿Qué hace de esta verdad algo presente? De acuerdo a la apocalíptica bíblica, lo que hace que esta verdad sea temporalmente relevante es su desarrollo y aparición en un tiempo especial de la historia humana: el tiempo del fin. Los libros de Daniel y Apocalipsis señalan que en este periodo el cronograma profético hallaría un cumplimiento mayor. Esta etapa de la historia se destaca por una concentración de acontecimientos de considerable magnitud afectando todo el curso de la humanidad. El primero fue la conmoción del cosmos, y en particular de nuestro planeta, con el famoso terremoto de Lisboa en el año 1755. ${ }^{1}$ Este evento marcó un antes y un después en todos los planos de la ciencia, filosofía y vida humana en general:

1 Cf. William H. Shea, “Cosmic Signs Through History”, Ministry, febrero 1999, 10-11.

https://doi.org/10.17162/recm.v19i1.1545 
Desde posiciones devotas, se justificaba el terremoto como un castigo divino, desde posiciones racionalistas, se intentaba comprender el seísmo aludiendo a motivos físicos y metafísicos, haciendo una lectura más allá de la tragedia. Muchos fueron los religiosos que aumentaron su influencia y alcanzaron gran fama a través de los púlpitos, destacando el padre Malagrida en Portugal; grandes pensadores como Voltaire, Rousseau o Kant vieron afectado su pensamiento por el terremoto, hasta tal punto que configuró sus cosmovisiones posteriores y generó un debate que pervivió durante mucho tiempo. Mantenemos que el seísmo agitó de una forma tan radical la conciencia ilustrada, cientificista, racional y optimista respecto al progreso de la humanidad y el conocimiento de la naturaleza, que supuso un punto de inflexión, un giro ineludible para entender el siglo XVIII. ${ }^{2}$

El segundo evento que conmocionó al mundo fue la Revolución Francesa hacia finales del siglo XVIII, acontecimiento que sentó las bases ideológicas de la sociedad moderna. Este evento también provocó un cisma en el poder religioso imperante hasta ese momento como fue la Iglesia Católica Romana (1798). ${ }^{3}$ Desde un punto de vista cultural, la revolución se desencadenó por un conjunto de factores ideológicos y sociales complejos que tuvieron en su fuerza antirreligiosa uno de sus vértices clave. ${ }^{4}$ Aquí yace una increíble paradoja: el ferviente anti-catolicismo de los revolucionarios

2 Ricardo Hurtado Simó, El ocaso del optimismo: De Leibniz a Hamacher. Debates tras el terremoto de Lisboa de 1755 (Madrid: Biblioteca Nueva, 2016), 15. Además, el autor añade: "A nuestro juicio, [el terremoto] originó en muchos sentidos un naciente existencialismo, similar al ocurrido en la Europa de entreguerras y con los campos de exterminio del siglo XX. Desde el terremoto de Lisboa, el mal irrumpe violentamente en la tierra y en la mentalidad ilustrada, provocando una reflexión profunda sobre la libertad humana, el lugar de Dios en el mundo, la temporalidad, la muerte y la contingencia del ser humano. Asimismo, la multitud de composiciones escritas (novelas, poemas, obras teatrales, cuentos...) y los centenares de cuadros y grabados sobre escenas del caos y las ruinas de Lisboa originaron una explosión cultural”. Ibíd.

3 “En febrero de 1798 el general Berthier ocupa la ciudad [Roma], depone a Pío VI y proclama la República romana. Las iglesias, los museos y el Vaticano fueron saqueados por los franceses, y el Papa era deportado a Valence, donde falleció el 29 de agosto de 1799, después de una larga y penosa peregrinación de año y medio hasta llegar a su destino, pasando por Siena, Florencia, Bolonia, Parma, Grenoble y Savona". Vicento P. Sancho, "Los Estados Pontificios desde la Revolución Francesa a los Pactos de Letrán (1798-1922)", Miscelánea Comillas 69, no. 134 (2011): 210.

4 Cf. Roger Chartier, The Cultural Origins of the French Revolution, trad. Lydia G. Cochrane (Durham, NC: Duke University Press, 1991). En especial el capitulo 5 titulado, "Dechristianization and Secularization", donde el autor analiza a fondo la interpretación de Alexis de Tocqueville sobre la actitud anticatólica de la Revolución Francesa. 
sucumbió ante la poderosa influencia que por siglos la iglesia ejerció sobre ellos. ${ }^{5}$ En otras palabras, que el rol de la Iglesia Católica Romana y su reinado de terror y persecución, con una religión basada en sus tradiciones estériles, en sus formas de gobierno tiránico, y sobre todo prohibiendo el acceso de la Biblia a las masas; produjo no solo una forma de religión decadente en la Europa de los siglos XVII y XVIII, mas bien consumaría en la revolución en Francia el fruto de una época de terror y caos. ${ }^{6}$

Finalmente, el tiempo del fin halla su centro en el comienzo de la segunda fase de la obra de expiación de Cristo en el santuario celestial en favor de su pueblo (Dn 8:14). Mientras diversos sucesos se desenvuelven en la arena humana, en los cielos se inicia en paralelo una obra de juicio (Ap 14:6) que busca restaurar a los seres humanos a la imagen de su Creador mediante la transformación del carácter y también restaurar las verdades salvíficas para lograr dicho objetivo supremo. ${ }^{7}$ Este es un punto importante a destacar. Por una parte, la restauración de la verdad sucede como respuesta a los siglos de ocultamiento de esta por parte del papado durante el Medioevo; por otra, que de esta manera el movimiento adventista es levantado por Dios como el único grupo continuador de la pureza del evangelio apostólico y su sentido de inminencia de la parusía, tal como lo refleja la siguiente ilustración: ${ }^{2}$

1) Paradigma protocristiano-apocalíptico 7) Paradigma escatológico adventista 2) Paradigma católico-romano medieval

3) Paradigma veteroeclesial helenista

4) Paradigma de la reforma protestante

5) Paradigma moderno ilustrado

6) Paradigma ecuménico contemporáneo (transmoderno)?

5 Cf. Dale K. Van Kley, The Religious Origins of the French Revolution: From Calvin to the Civil Constitution, 1560-1791 (New Haven: Yale University Press, 1996).

6 Esta es también la interpretación de Ellen G. White en el capitulo 16 de su obra El gran conflicto, "La Biblia y la Revolución Francesa".

7 El concepto hebreo envuelto en la expresión נצדק de Daniel 8:14 implica la idea de que algo es "purificado", "vindicado", "restaurado". A su vez, tiene a Levítico 16 como trasfondo ideológico y reviste implicancias no solo teológicas sino también antropológicas, poco exploradas en la teología adventista reciente. Para algunas consideraciones sobre la antropología del libro de Daniel, vease Allan Bornapé, "Image and Degradation as a Philosophy of History in Daniel 2", TheoRhema 16, no. 1 (2021): 7-32.

8 José M. Alarcón, “Hacia una critica de la razón misionológica: En busca del sentido originario de misión", Advenimiento (2004): 34. Véase también Hans Kung, El cristianismo: Esencia e historia (Madrid: Trotta, 1996), i. Alarcón ha actualizado este esquema 
Por lo tanto, el factor de tiempo para la verdad presente está definido por situarse en el último período de la historia, el tiempo del fin, y porque el modelo del santuario celestial nos dirige hacia Cristo y su obra como el centro de gravedad teológico del adventismo donde todas las principales enseñanzas bíblicas se reúnen y hallan su sentido pleno.

\section{Verdad presente: Conocimiento}

Tras los significativos acontecimientos proféticos que sucedieron desde mediados del siglo XVIII en adelante, el evento de Cristo en el santuario celestial en 1844 abrió un tesoro de verdades con un nuevo brillo no visto hasta ese entonces. El texto de Daniel 8:14, que es considerado el fundamento de nuestra fe, reveló junto con Daniel 7 la realidad de un juicio en los cielos en favor del pueblo de Dios (Dn 7:22). Esto dio paso a una comprensión mucho mas completa sobre las nociones de la ley de Dios, el pecado y la salvación del hombre. ${ }^{9}$ Si solo pensamos en la relación estrecha entre los capítulos 7 , 8 y 9 de Daniel; los tres tratan con el concepto central de la צדקה, la "justicia" de Dios, ${ }^{10}$ y tienen como su foco a Dios en su santuario y al pueblo de Dios. ${ }^{11}$ Entonces, Daniel 9 es la base soteriológica de estos pasajes porque es donde se inicia el proceso de expiación en la historia puesto que el Mesías trae consigo una צדק עלמים, "justicia eterna (o perdurable)". ${ }^{12}$ Por lo tanto, Cristo

de paradigmas históricos, pero su propuesta se hace desde el punto de vista de la escatología adventista.

9 Veanse, por ejemplo, los aportes eruditos de Roy Gane sobre los significados y diferencias entre las impurezas rituales (mortalidad para Jacob Milgrom o naturaleza caída en el lenguage teológico adventista) y las faltas morales (pecado en el lenguaje bíblico y adventista). Roy Gane, Cult and Character. Purifrications Offerings, Day of Atonement, and Theodicy (Winona Lake, IN: Eisenbrauns, 2005), 123-133. En cuanto al concepto de la ley de Dios, véase Roy Gane, Old Testament Law for Christians. Original Context and Enduring Application (Grand Rapids: Baker Academic, 2017).

10 Para ver los diversos paralelos lingüísticos entre Daniel 8 y 9, véase John Goldingay, Daniel, WBC 30, rev. ed. (Grand Rapids: Zondervan, 2019), 486-487.

11 Jacques Doukhan, "The Seventy Weeks of Dan 9: An Exegetical Study", AUSS 17, no. 1 (1979): 10. El mismo binomio santuario y pueblo es el foco de Daniel 7 y 8.

12 Al constituirse en la respuesta a la oración de Daniel, los textos de Daniel 9:24-27 retratan al Mesías trayendo una justicia que va más allá de la situación inmediata del profeta. Nótese que la expresión צדק עלמים, "justicia eterna", se erige aquí, en nuestra

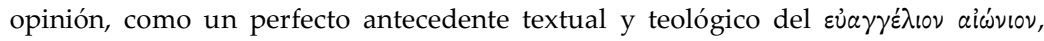
"evangelio eterno", de Apocalipsis 14:6. En ambos capítulos, las expresiones son happax legomenon. 
como el "Hijo del Hombre" y Mesías con su sacrificio y funciones sacerdotales nos mostró también una rica cristología. ${ }^{13}$ Así pues, puede afirmarse que la redención del ser humano consiste en el sacrificio de Cristo y el juicio antes de su segunda venida, siendo el primero base del segundo, lo que puede esquematizarse de la siguiente manera:

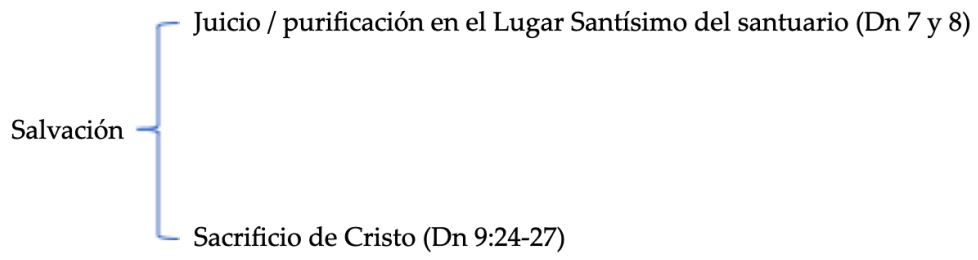

Por otro lado, el libro de Daniel en su ultimo capitulo predice que "la ciencia aumentaría" (12:4), refiriéndose con ello a que las palabras selladas de las profecías finales (Dn 7-12), serian abiertas tal como Apocalipsis lo confirma (Ap 10). Este aumento del conocimiento, que apunta a la naturaleza progresiva de la revelación y comprensión del conocimiento salvífico en la historia (como una gran síntesis teológica). ${ }^{14}$ Como muestra de esto es que gracias al genero apocalíptico de los libros de Daniel y Apocalipsis, nuestros pioneros llegaron a una organización de las verdades descubiertas alcanzando tempranamente un sentido de un "sistema" teológico articulador. ${ }^{15}$

Sin embargo, durante esta época aparecieron diferentes corrientes filosóficas que marcaron gran parte de los siglos XIX en adelante. Póngase atención a las fechas. Comenzando por la obra de Julius Welhaussen (18441918) y su obra Prolegomena to the History of Israel (1878), en la que se propone un nuevo(s) autor(es) y datación para Levítico (el manual del santua-

13 Cf. Arthur J. Ferch, The Son of Man in Daniel 7 (PhD Dissertation, Andrews University, 1983).

14 Para esta temática, véase Willhem Van Gemeren, The Progress of Redemption: The Story of Salvation from Creation to the New Jerusalem (Grand Rapids: Baker, 1995).

15 Para estas ideas, véase Alberto Timm, El santuario y los mensajes de los tres ángeles (Florida: Asociación Casa Editora Sudamericana, 2018). Los únicos libros a mi conocimiento que han abordado el tema de la verdad presente como un importante concepto teológico articulador son Reinder Bruinsma, Present Truth Revisited: An Adventist Perspective on Postmodernism (Netherlands: Ut Intelligam, 2014); y también Miguel A. Nuñez, La verdad progresiva: Desarrollo historico de la teologia adventista, 6ta. ed. (Valencia: Fortaleza ediciones, 2018). Sin embargo, estas obras tienen interpretaciones críticas altamente insatisfactorias.

https://doi.org/10.17162/recm.v19i1.1545 
rio en la Biblia), y se comenzó a interpretar la religión de Israel como cualquier otra religión pagana. ${ }^{16}$ La publicación de El origen de las especies de Charles Darwin (1859), definió la visión filosófica tanto de la ciencia como de la concepción de la vida en general. ${ }^{17}$ Las ideas filosóficas, políticas y económicas de Karl Marx (1818-1883), tuvieron un notable impacto en pensadores posteriores ${ }^{18}$ Lo mismo puede decirse de los destacados filósofos G. W. F. Hegel (1770-1831), Immanuel Kant (1724-1804) y Friedrich Nietzsche (1844-1900), entre otros (la lista es larga), quienes desde distintas perspectivas reconfiguraron por completo el paisaje del pensamiento occidental.

Otra figura, entre tantas, es la de Sigmund Freud (1856-1939), padre del psicoanálisis y quien realizó un completo replanteamiento de la sexualidad humana. En el plano de la religión, algunos de los principales movimientos modernos surgieron durante este periodo. ${ }^{19}$ El espiritismo con las hermanas Fox (1848) Helena Blavatsky (1831-1891) y la Teosofía, o la figura de Allan Kardec (1804-1869), son solo algunos pocos ejemplos del profundo despertar y efervescencia religiosos que circundó al "amanecer del adventismo" ${ }^{20}$

16 Gerhard Hasel, Old Testament Theology. Basic Issues in the Current Debate (Grand Rapids: Eerdmans, 1991), 23

17 John Baldwin observó agudamente lo siguiente: “Hacia finales de julio de 1844, Darwin completó su manuscrito de 189 paginas que resumía su teoría de las especies. El expandió la versión que había preparado dos años antes. Los eruditos se refieren a este como el boceto de Darwin de 1844. Similar a su posterior libro El origen de las especies, el ensayo propuso el desarrollo de las especies biológicas a través de un proceso evolucionario comprendiendo millones de años. Sin embargo, de acuerdo a la interpretación historicista-apocalíptica, Dios levantó durante el otoño de 1844 en Norteamérica una proclamación inicial del mensaje del primer ángel de Apocalipsis 14 como una verdad presente que podría dirigirse al mundo entero. Entonces, cuando Darwin en su manuscrito de 1844 sugiere aquellas formas biológicas desarrolladas por sobre millones de años, Dios en el mismo año envió un mensaje especial al mundo de que Él creó las formas de vida básicas en seis días, no en millones de años", John Baldwin, "Revelation 14:7: An Angel's Worldview," en Creation, Catastrophe and Calvary: Why a Global Flood Is Vital to the Doctrine of Atonement (Hagerstown, MD: Review \& Herald, 2000), 25.

18 Principalmente su obra El capital.

19 Mormonismo (1830), Testigos de Jehová (1870), Pentecostalismo (1906), y muchos otros.

20 Haciéndome eco del bello titulo del libro de Aecio Cairus, El amanecer del advenimiento: La eclosión que cautivó al mundo cristiano (Libertador San Martin, Argentina: Editorial Universidad Adventista del Plata, 2016).

https://doi.org/10.17162/recm.v19i1.1545 
Desde la cosmovisión del gran conflicto, todo este periodo histórico es permeado de manera transversal por el escepticismo y el panteísmo, ${ }^{21}$ forjando una contra-espiritualidad que no hace sino socavar y/o anular la influencia del mensaje de salvación proveniente del santuario y su impacto histórico para la ética cristiana. ${ }^{22}$

Por lo tanto, al volver al tema del santuario y sus verdades, el conocimiento acerca de Cristo y su obra de salvación en el santuario celestial permitió una comprensión mucho mas profunda y abarcante de las principales verdades bíblicas o pilares de la fe como aquellos cimientos de todo el edificio teológico de las Escrituras, teniendo como su modelo arquitectónico el santuario. Así, la comprensión de la ley de Dios y el sábado, el don profético, la inmortalidad condicional del alma, la reforma pro salud, el juicio investigador y la segunda venida de Cristo encuentran en el santuario un punto de encuentro en común. ${ }^{23} \mathrm{La}$ apertura del santuario (y en particular del Lugar Santísimo) resultó ser un enorme desafío para la iglesia debido a que impulsó una investigación teológica integradora, tanto del conocimiento de las Escrituras como también de la misión de la iglesia. ${ }^{24}$ Sin embargo, la influencia del mundo teológico académico (católico y protestante $)^{25}$ desde mediados del siglo XX en adelante ha generado una sostenida influencia en nuestra comprensión teológica adventista, fraccionando la posibilidad de un sistema teológico unificado, ${ }^{26}$ y como consecuencia, un

21 Paul Ricoeur llama a Nietzsche, Marx y Freud los "maestros de la sospecha". Paul Ricoeur, Freud: Una interpretación de la cultura (México: Siglo XXI, 1970), 32-35. Cf. Fernando A. Fraga, "La escatología secular contemporánea: ¿Retorno a la inmanencia?", DavarLogos 3, no. 1 (2004): 37-55.

22 Para un fino y profundo análisis de la historia de las ideas filosóficas desde la cosmovisión del gran conflicto y su impacto en la filosofía, teología cristiana y el adventismo, véase Carsten Johnsen, The Mystic Omega of Endtime Crisis (Francia: Untold Story Publishers, 1982).

23 Cf. Fernando Canale, "From Vision to System: Finishing the Task of Adventist Theology - Part III: Sanctuary and Hermeneutics", JATS 17, no. 2 (2006): 36-80.

24 Véase ahora el detallado estudio de Adrian Petre, "The Three Angels' Messages as the Teleological Principle of the Adventist Theological System", en Eschatology from an Adventist Perspective: Proceedings of the Fourth International Bible Conference, Rome, June 11-20, 2018, ed. Elias Brasil de Souza (Silver Spring, MD: Biblical Research Institute, 2021), 429-455.

25 Fernando Canale, "The Eclipse of Scripture and the Protestantization of the Adventist Mind - Part 2: From the Evangelical Gospel to Culture", JATS 22, no. 1 (2011): 102133.

26 Preocupados en una exégesis y teología academicista, crítica y vanguardista, muchos teólogos adventistas en la actualidad están más preocupados por desarrollar una excesiva especialización e implementación de nuevos métodos de exégesis, provocando 
sentido de misión diverso y a veces confuso. ${ }^{27}$ En cambio, todo este conocimiento que se abrió desde el santuario y que se encuentra condensado en el mensaje de los tres ángeles de Apocalipsis 14:6-12, ${ }^{28}$ fue otorgado con el elevado propósito de preparar a un pueblo para una misión especial:

La verdad presente en Apocalipsis 14:6-12

\begin{tabular}{|c|c|}
\hline $\begin{array}{c}\text { Evangelio eterno } \\
\text { (Ap 14:6) } \\
\text { Mensaje del primer ángel } \\
\text { (Ap 14:6, 7) }\end{array}$ & Santuario - Don profético ${ }^{29}$ \\
$\begin{array}{c}\text { Mensaje del segundo ángel } \\
\text { (Ap 14:8) }\end{array}$ & Ley de Dios - Juicio - Sábado \\
$\begin{array}{c}\text { Mensaje del tercer ángel } \\
\text { (Ap 14:9-12) }\end{array}$ & Inmortalidad condicional del alma \\
Sábado - Reforma pro salud \\
\hline \multicolumn{2}{|c|}{$\begin{array}{c}\text { Segunda venida de Cristo } \\
\text { (Ap 14:13-20) }\end{array}$} \\
\hline
\end{tabular}

una separación rígida entre las distintas disciplinas de la teología. Como consecuencia de todo esto, existe una labor teológica y pastoral aislada los unos de los otros, sin posibilidad de trabajar juntos con un mismo propósito. Creemos que, si tomáramos seriamente la naturaleza integradora del santuario y sus grandes verdades, nuestro quehacer teológico llegaría a ser uno de unidad disciplinar y misionera. En suma, la teología del santuario debe ser una teología en comunidad. Cf. Carsten Johnsen, The Mystic Omega of Endtime Crisis (Francia: Untold Story Publishers, 1982).

27 Considero que las palabras de Fernando Canale son cruciales: "Ser-en-la-misión requiere que los adventistas exalten al Cristo de la Biblia al experimentar y compartir el sistema completo y armonioso de filosofía y teología con la iglesia y el mundo. Este mensaje/sistema (los mensajes de los tres ángeles, la verdad presente, la plataforma de la verdad) tiene poder porque incluye las doctrinas y la información de la Escritura en una metanarrativa coherente, que todo lo abarca, centrada en Cristo. En esta metanarrativa, la cruz es el centro de atracción, y el Cristo encarnado, resucitado y que ministra en el santuario celestial es el centro de la espiritualidad y la salvación". Fernando Canale, "El mensaje y la misión del remanente: Un enfoque metodológico", en Mensaje, misión y unidad de la iglesia, ed. Ángel M. Rodríguez (Florida: Asociación Casa Editora Sudamericana, 2015), 276-277.

28 Véase la excelente síntesis de Alberto R. Timm, "The Prophetic Nature of Adventism", en The Word: Searching, Living, Teaching, ed. Artur Stele (Silver Spring, MD: Review \& Herald, 2015), 230-242.

29 El don profético lo situamos al principio de los mensajes de los tres ángeles junto al santuario porque fue otorgado a la iglesia remanente desde su origen histórico, particularmente en el tiempo del fin como la manera de Dios de conducir en forma segura a su pueblo y preservar su verdad. Véase al respecto, Herbert Douglass, Mensajera del Señor: El ministerio profético de Elena G. de White (Nampa, ID: Pacific Press, 2000), 268-277.

https://doi.org/10.17162/recm.v19i1.1545 
En palabras de Ellen G. White:

La Biblia ha acumulado y reunido sus tesoros para esta última generación. Todos los grandes eventos y las solemnes transacciones de la historia del Antiguo Testamento, han sido repetidas y se están repitiendo en la iglesia en estos últimos días.... Allí todas las verdades acumuladas se nos presentan con fuerza para que aprovechemos sus enseñanzas. Estamos bajo la influencia de todas ellas. ¿Qué clase de personas debemos ser nosotros, a quienes ha sido dada toda esta rica luz como herencia? $\mathrm{Al}$ concentrarse toda la influencia del pasado con una luz nueva y acrecentada del presente, a todos los que la siguen se les da un poder intensificado. Su fe aumentará y se pondrá en ejercicio en el tiempo presente, despertando una energía y un fervor intensamente ampliado; y en base a una dependencia de Dios y de su poder, llenarán el mundo y enviarán la luz del Sol de Justicia hasta los confines de la tierra. ${ }^{30}$

El elevado estatus espiritual del remanente del tiempo del fin es representado bien en el simbolismo de los 144000 (Ap 7 y 14), quienes antes de proclamar el último mensaje de amonestación al mundo (Ap 14:6-12), son previamente descritos como un grupo que en medio de una crisis global (Ap 13) han sido transformados por el conocimiento de la verdad presente, y que reflejararán el carácter de Jesucristo en sus vidas para "alumbrar la tierra con su gloria" (Ap 18:1). ${ }^{31}$

\section{Fin último de la verdad presente: la restauración de la imagen/carácter de Dios en la humanidad}

Toda esta reflexión nos lleva finalmente a la razón de ser de la iglesia: la proclamación del evangelio. Esta misión se encuentra fundamentada en un mensaje preciso y oportuno en la historia, a saber, el tiempo del fin. Por lo

Ellen G. White, Mensajes selectos (Doral, FL: Asociación Publicadora Interamericana, 200), 3:387.

31 “El aceite, tan necesario para los que están representados por las vírgenes necias, no es algo que deba ser dejado de lado. El creyente debe traerlo al santuario de su ser para que lo limpie, lo refine y lo santifique. No es teoría lo que se necesita; son las sagradas enseñanzas de la Biblia, las que no constituyen doctrinas inciertas y sin sentido sino verdades vitales que comprometen intereses eternos centrados en Jesús. En él reside todo el sistema de verdades divinas. La salvación del creyente, mediante la fe en Cristo, es el pilar fundamental de la verdad", Ellen G. White, Recibiréis poder (Florida: Asociación Casa Publicadora Sudamericana, 2009), 18.

https://doi.org/10.17162/recm.v19i1.1545 
tanto, es un mensaje que abarca la totalidad de la verdad bíblica revelando su poder para restaurar la imagen de Dios en los seres humanos:

El tema central de la Biblia, el tema alrededor del cual se agrupan todos los demás del Libro, es el plan de la redención, la restauración de la imagen de Dios en el alma humana. Desde la primera insinuación de esperanza que se hizo en la sentencia pronunciada en el Edén, hasta la gloriosa promesa del Apocalipsis: "Y verán su rostro, y su nombre estará en sus frentes", el propósito de cada libro y pasaje de la Biblia es el desarrollo de este maravilloso tema: La elevación del hombre, el poder de Dios, "que nos da la victoria por medio de nuestro Señor Jesucristo". El que capta este pensamiento, tiene ante sí un campo infinito de estudio. Tiene la llave que le abrirá el tesoro de la Palabra de Dios. ${ }^{32}$

En efecto, el concepto de carácter ${ }^{33}$ y su restauración es una pieza central a lo largo de las Sagradas Escrituras. ${ }^{34} \mathrm{Si}$ uno piensa solamente en personajes legendarios como Enoc, que caminó con Dios y fue llevado al cielo; y

32 Ellen G. White, La educación (Florida: Asociación Casa Publicadora Sudamericana, 1998), 125.

33 La palabra carácter etimológicamente se conecta con dos raíces: el término griego ethos (carácter) y el término latín mores (costumbre). Este último se remite al primero, ya que son las costumbres y hábitos los que van formando el carácter a lo largo de la vida. Ana Escribar Wicks, Ética narrativa: Antecedentes y posibles aportes al juicio moral (Santiago: Ediciones Universidad Diego Portales, 2013), 21. Para los diversos matices de la palabra carácter como "costumbres, hábitos, pensamiento, etc.", véase Pierre Chantraine, "El" $\omega \theta \alpha$ ", Dictionnaire étymologique de la langue grecque (Paris, Éditions Klincksieck, 1999), 327. Para una conceptualización inicial en el AT, Elaine A. Goodfriend observa que la frase el temor de Jehova es una de las claves para entender el concepto de carácter moral en la Biblia Hebrea. Elaine A. Goodfriend, "Ethical Theory and Practice in the Hebrew Bible", en The Oxford Handbook of Jewish Ethics and Morality, ed. Elliot N. Dorff y Jonathan K. Crane (New York: Oxford University Press, 2013), 35-50. De forma similar, Anne W. Stewart, Poetic Ethics in Proverbs: Wisdom Literature and the Shaping of the Moral Self (New York: Cambridge University Press, 2015); Daniel Block, "The First Principle of Wisdom in Deuteronomy: The Fear of YHWH as Allegiance to YHWH Alone," en Interpreting the Old Testament Theologically: Essays in Honor of Willem A. VanGemeren, ed. Andrew T. Abernethy (Grand Rapids: Zondervan, 2018), 150-164.

34 La centralidad del concepto de carácter en los escritos de Ellen G. White es clara. Sin embargo, muy pocos estudios han sido escritos por teólogos adventistas acerca de este concepto en el texto bíblico. Esta es una deuda pendiente y una valiosa oportunidad de estudio. Para una síntesis académica actual, véase John Barton, "Ethics and Character Formation in Biblical Wisdom Texts", en Seeking Wisdom's Depths and Torah's Heights. Essays in Honor of Samuel E. Balentine, ed. Barry R. Huff y Patricia Vesely (Macon, GA: Smyth \& Helwys, 2020), 232-246. Puede también consultarse la bibliografía de la nota de pie de página anterior.

https://doi.org/10.17162/recm.v19i1.1545 
Noé, de quien se dijo que era "varón justo, perfecto en sus generaciones" (Gn 6:9); vemos que la descripción del carácter de cada uno de ellos es enfatizada. Lo mismo sucede con los demás patriarcas y el resto de personajes descritos en el AT, que en su naturaleza biográfica de la historia e inspirada, retrata con sus altos y bajos ejemplos de vida, y que en ultima instancia apuntaban hacia el Mesías prometido. Los reyes de Israel, por ejemplo, fueron evaluados moralmente por el modelo del rey David el cual, a su vez, en realidad señalaba hacia Cristo, el antitípico “Hijo de David" (Mt 1:1; Ap 5:5; 22:16). ${ }^{35}$

En el NT, sin embargo, vemos un cuadro más elaborado del tema del carácter y su desarrollo. En los Evangelios ya aparece el concepto de justificación por la fe, ${ }^{36}$ y el concepto del discipulado cristiano que puede interpretarse como el desarrollo del carácter de los discípulos. ${ }^{37}$ En los escritos de Pablo, la metáfora del templo para el cristiano individual y también para la comunidad de creyentes (imagen ya anticipada en la propia persona de Jesús), ${ }^{38}$ es un fiel reflejo de la alta visión del apóstol de una iglesia santa y pura donde el Espíritu Santo haga su morada. ${ }^{39}$ El apóstol Pedro invita a los cristianos a ser "participantes de la naturaleza divina" (2 P 1:3-8). Así, quienes adoptan la escalera de las virtudes allí descritas, desarrollarán progresivamente un carácter semejante al de Cristo, quien es el mayor ejemplo

Cf. Amos Frish, "Comparison with David as a Means of Evaluating Character in the Book of Kings", JHebS 11 (2011): 1-20; Alison L. Joseph, Portrait of the Kings: The Davidic Prototype in Deuteronomistic Poetics (Philadelphia: Fortress, 2015).

36 Clinton Wahlen, "Justification in the Gospels", JATS 24, no. 1 (2013): 42-53.

37 Cf. Sookgoo Shin, Ethics in the Gospel of John Discipleship as Moral Progress (Leiden: Brill, 2019).

38 James Sweeney, "Jesus, Paul, and the Temple: An Exploration of Some Patterns of Continuity", JETS 46, no. 4 (2003): 605-631.

39 Eyal Regev, "Community as Temple: Revisiting Cultic Metaphors in Qumran and the New Testament", BBR 28, no. 4 (2018): 604-631; y especialmente el excelente libro de Hannah K. Harrington, The Purity and Sanctuary of the Body in Second Temple Judaism. Journal of Ancient Judaism Supplements 3 (Göttingen: Vandenhoeck \& Ruprecht, 2019). Esta dimensión antropológica del estudio del santuario casi no ha recibido atención en la teología adventista. Creemos que la principal razón del descuido de este importante tópico se debe al impacto de la apostasía de John H. Kellog y su interpretación panteísta del cuerpo como un "templo del alma", tal como lo advirtió perspicazmente el historiador C. Mervyn Maxwell, "Sanctuary and Atonement in SDA Theology: An Historical Survey", en The Sanctuary and the Atonement: Biblical, Historical, and Theological Studies, ed. Arnold V. Wallenkampf y W. Richard Lesher (Washington, DC: Review \& Herald, 1981), 519.

https://doi.org/10.17162/recm.v19i1.1545 
de vida, cumpliendo de esta manera el más grande anhelo de la iglesia cristiana. ${ }^{40}$ Por ultimo, los libros de Daniel y Apocalipsis, con su fuerte foco en el fin de la historia, presentan el cuadro final de la perfección del carácter del pueblo remanente de Dios. ${ }^{41}$

La idea importante del carácter cristiano para el pensamiento de Ellen G. White representa un concepto antropológico multidimensional que es transversal en toda su obra y que es central no solo para el sistema de educación adventista, sino también para el plan de redención y destino del hombre. John Fowler lo sintetiza de la siguiente manera: Primero, la importancia del carácter puede observarse desde el punto de vista de la historia divina. Ellen $\mathrm{G}$. White sostiene que la historia comprende un conflicto entre el bien y el mal bajo el liderazgo de Cristo y Satanás, respectivamente. En ese contexto macro, se requiere la vindicación de Dios a través de la restauración y perfección del carácter del ser humano. Segundo, el carácter es visto como la prueba final de la validez y confiabilidad de la fe cristiana para alcanzar como su meta la semejanza al carácter de Dios. Tercero, el carácter es esencial para la realización del potencial de vida, así como para el cumplimiento del destino del ser humano en una vida más allá del presente, a saber, la eternidad. ${ }^{42}$ Un posible esquema de todo esto puede graficarse de la siguiente manera:

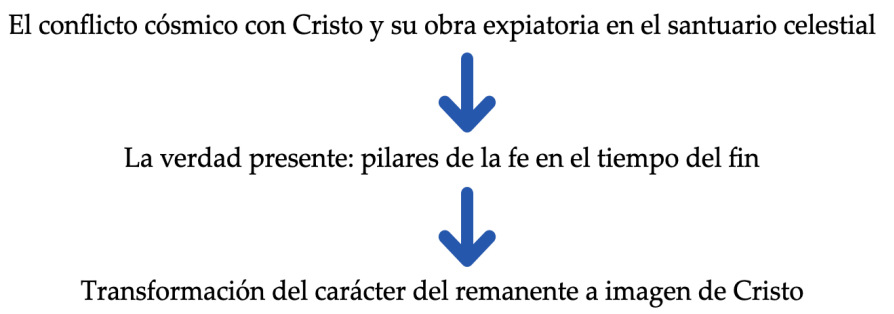

40 Cf. Andreas J. Kostenberger, Handbook on Hebrews through Revelation (Grand Rapids: Baker Academic, 2020), 156-158.

41 Renel Gomez, “El pueblo de Dios de Levítico como 'las primicias de la tierra' a los 144.000 en Apocalipsis", Advenimiento 7, no. 1 (2019): 32-41. Sobre el concepto del carácter, véase Beatrice Neall, The Concept of Character in the Apocalypse with Implications for Character Education (Washington, DC: University Press of America, 1983); Larry Lichtenwalter, "Worship, Eschatology, and Ethics: The Revelation of John and the Worshiping Imagination", en Meeting with God on the Mountains: Essays in Honor of Richard M. Davidson, ed. Jiří Moskala et al. (Berrien Springs, MI: Seventh-day Adventist Theological Seminary, Old Testament Department, 2016), 465-501.

42 John Fowler, The Concept of Character Development in the Writings of Ellen G. White (PhD Dissertation, Andrews University, 1977), 222-223.

https://doi.org/10.17162/recm.v19i1.1545 
Finalmente, esta obra de restauración de la imagen o carácter divino en la humanidad recibe también el nombre de justificación por la fe descrito claramente en el evangelio eterno y que en el último libro de la Biblia halla su consumación. ${ }^{43}$ Este último mensaje de esperanza al mundo, como lo presenta el mensaje del tercer ángel de Apocalipsis 14:9-11, es un fuerte llamado a no adorar bajo el sistema de religión papal, aceptando su falso día de reposo (domingo), para no recibir su marca. Todos estos actos, la adoración falsa y la recepción de la marca de la bestia responden a propósitos satánicos con el propósito de transformar a los moradores de la tierra a su propia imagen. ${ }^{44}$ Mediante sus obras maestras, la idolatría y el espiritismo (descrito como el vino de Babilonia), ${ }^{45}$ Satanás intentará neutralizar la verdad presente presentando al mundo un falso evangelio. En medio de este conflicto de adoración, el mensaje final que la iglesia debe anunciar, la verdad presente, es una invitación directa a volver al Creador y su baluarte de verdad - aceptación y observancia del sábado bíblico - y así recibir su sello de aprobación y protección (Ap 7:1-3; Ap 14:6-12; cf. Ez 9).

\section{Conclusiones}

La idea de una verdad presente es una noción amplia, profunda y de suma importancia para la iglesia de hoy. Nació como fruto del cumplimiento profético, abrió una inmensa fuente de conocimiento bíblico y sobre todo definió cuál es el mensaje de salvación para el tiempo del fin. Es un legado inestimable y que reviste un sentido de urgencia, el cual Dios ha dejado en nuestras manos. Nuestra misión consiste en vivir el mensaje y proclamarlo. Oremos con fervor al Señor para ser un pueblo digno de sus verdades y para seguir el consejo inspirado:

Son muchas las preciosas verdades que contiene la Palabra de Dios, pero es "la verdad presente" lo que el rebaño necesita. He visto el peligro que existe de que los mensajeros se desvíen de los puntos importantes de la

43 Entiéndase la justificación como justicia imputada e impartida, por lo tanto, unida también al proceso de la santificación cristiana. Roy Gane, "Legal Substitution and Experiential Transformation in the Typology of Leviticus", Ministry, noviembre 2013, 18-21; Clinton Wahlen, "The Letter to Laodicea and the Eschatology of Revelation", JATS 29, no. 1 (2018):138-148.

44 Nótese el uso de Génesis 1:27 y 2:7 en Apocalipsis 13:14, 15 (cf. Dn 3). Para un completo tratamiento de este tópico, véase Rebekah Yi Liu, The Background and Meaning of the Image of the Beast in Rev. 13:14, 15 (PhD Dissertation, Andrews University, 2016).

45 Ekkehardt Mueller, "Babylon Identified", en The Word: Searching, Living, Teaching, ed. Artur Stele (Silver Spring, MD: Review \& Herald, 2015), 169.

https://doi.org/10.17162/recm.v19i1.1545 
verdad presente para espaciarse en temas que no tienden a unir el rebaño ni santificar el alma. En esto, Satanás aprovechará toda ventaja posible para perjudicar la causa. ${ }^{46}$

46 Ellen G. White, Primeros escritos (Mountain View, CA: Asociación Publicadora Interamericana, 1962), 63.

https://doi.org/10.17162/recm.v19i1.1545 